\title{
Fully Discrete Orthogonal Collocation Method of Sobolev Equations
}

\author{
Ning Ma, Wenliang Bian, Xiaofei Lu \\ College of Science, China University of Petroleum, Beijing, China \\ Email: bjmaning@163.com
}

How to cite this paper: Ma, N., Bian, W.L. and Lu, X.F. (2017) Fully Discrete Orthogonal Collocation Method of Sobolev Equations. Journal of Applied Mathematics and Physics, 5, 2354-2359. https://doi.org/10.4236/jamp.2017.512192

Received: November 21, 2017 Accepted: December 24, 2017 Published: December 27, 2017

Copyright () 2017 by authors and Scientific Research Publishing Inc. This work is licensed under the Creative Commons Attribution International License (CC BY 4.0).

http://creativecommons.org/licenses/by/4.0/

\begin{abstract}
In this paper, the fully discrete orthogonal collocation method for Sobolev equations is considered, and the equivalence for discrete Garlerkin method is proved. Optimal order error estimate is obtained.
\end{abstract}

\section{Keywords}

Sobolev Equations, Orthogonal Collocation Method, Error Estimate

\section{Introduction}

Sobolev equations are a class of mathematical physics equations, which are widely used in engineering field. Many numerical methods have been proposed, such as the characteristic difference method [1], the $H^{\mathrm{t}}$-Galerkin Finite Element Method [2], the mixed finite element [3] and so on. The collocation method now is widely used in many fields including engineering technology and computational mathematics. Many applications have been proved effectively, e.g. the heat conduction equation [4], stochastic PDEs [5] and reaction diffusion equation [6]. The collocation method has high convergence order and does not need to calculate numerical integration so that the calculation is simple. So now we consider the application of fully discrete collocation method for Sobolev equations. We consider the linear Sobolev equations as follows:

$$
\left\{\begin{array}{l}
u_{t}=\nabla\left(a \nabla u_{t}+b \nabla u\right)+f(x, y, t),(x, y) \in \Omega, t \in(0, T], \\
\left.u\right|_{\partial \Omega}=0,(x, y) \in \partial \Omega, t \in[0, T], \\
\left.u\right|_{t=0}=u_{0}(x, y),(x, y) \in \Omega .
\end{array}\right.
$$

In the equations, $u_{t}$ is the time derivative of $u$, and $\nabla u$ is the gradient of $u$. $\Omega=[0,1] \times[0,1], \partial \Omega$ is the border of $\Omega . a=a(x, y, t)$ and $b=b(x, y, t)$ are known bounded differentiable functions. 


\section{Fully-Discrete Collocation Method}

First, time is divided into $n$ equal parts. Let $\Delta t=\frac{T}{n}$ be the time step. Then we introduce the following notations:

$$
t_{n}=n \Delta t, u^{n}=u\left(t_{n}\right), \partial_{t} u^{n}=\frac{u^{n}-u^{n-1}}{\Delta t}, \nabla u^{n}=\nabla u\left(t_{n}\right), \partial_{t} \nabla u^{n}=\frac{\nabla u^{n}-\nabla u^{n-1}}{\Delta t} .
$$

Then we discrete the spatial region $\Omega$ into grids by points $\left(x_{i}, y_{j}\right), i=0,1,2, \cdots, M, j=0,1,2, \cdots, N$ and $x_{i}, y_{j}$ are satisfied $0=x_{0}<x_{1}<\cdots<x_{M}=1,0=y_{0}<y_{1}<\cdots<y_{N}=1$. Let [7]

$$
\begin{aligned}
& \Omega_{i j}=\left[x_{i-1}, x_{i}\right] \times\left[y_{j-1}, y_{j}\right], h_{x_{i}}=x_{i}-x_{i-1}, h_{y_{j}}=y_{j}-y_{j-1}, h=\max \left\{h_{x}, h_{y}\right\}, \\
& H_{3}=\left\{v=v(x, y) \in C^{1}(\Omega)|v|_{\Omega_{i j}} \text { is a Bi-cubic Hermit polynomial }\right\}, \\
& H_{3}^{0}=\left\{v=v(x, y) \in H_{3}|v|_{\partial \Omega}=0\right\} .
\end{aligned}
$$

The four Gauss points $\left(x_{i k}, y_{j l}\right) ; k, l=1,2$ in $\Omega_{i j}$ are collocation points as follows: $x_{i k}=x_{i-1}+h_{x_{i}} \theta_{k}, y_{j l}=y_{j-1}+h_{y_{j}} \theta_{l}, k, l=1,2$, where $\theta_{1}=(3-\sqrt{3}) / 6$, $\theta_{2}=(3+\sqrt{3}) / 6$. Then the intermediate variable $q=a \nabla u_{t}+b \nabla u$ is introduced so that the orthogonal collocation scheme as follows can be established. Seeking $(U, Q):[0, T] \rightarrow H_{3} \times H_{3}$, such that

$$
\begin{gathered}
\left\{\partial_{t} U^{n}-\nabla Q^{n}-f^{n}\right\}\left(x_{i k}, y_{j l}\right)=0, \\
\left\{Q^{n}-\left(a^{n} \partial_{t} \nabla U^{n}+b^{n} \nabla U^{n}\right)\right\}\left(x_{i k}, y_{j l}\right)=0, \\
\left\{\begin{array}{l}
\left.U\right|_{\partial \Omega}=0, t \in[0, T], \\
\left.U\right|_{t=0}=u_{0}(x, y) .
\end{array}\right.
\end{gathered}
$$

Now we set the following notations [4]:

$$
\begin{aligned}
& \langle u, v\rangle=\sum_{i=1}^{M} \sum_{j=1}^{N}\langle u, v\rangle_{i j}=\sum_{i=1}^{M} \sum_{j=1}^{N} \frac{h_{x} h_{y}}{4} \sum_{k, l=1}^{2} u v\left(x_{i k}, y_{j l}\right), \\
& \langle u, v\rangle_{x}=\sum_{i=1}^{M}\langle u, v\rangle_{i x}=\sum_{i=1}^{M} \frac{h_{x}}{2} \sum_{k=1}^{2} u v\left(x_{i k}, y\right), \\
& \langle u, v\rangle_{y}=\sum_{j=1}^{N}\langle u, v\rangle_{j y}=\sum_{j=1}^{N} \frac{h_{y}}{2} \sum_{l=1}^{2} u v\left(x, y_{j l}\right), \\
& \|u\|_{i}^{2}=\langle u, u\rangle_{i},\|u\|^{2}=\langle u, u\rangle .
\end{aligned}
$$

Next, we are going to prove existence and uniqueness of collocation solution and obtain the error estimate.

\section{Discrete Galerkin Method}

Consider the following discrete Galerkin scheme

$$
\left\{\begin{array}{l}
\left\langle\partial_{t} U^{n}, z_{1}\right\rangle+\left\langle\nabla Q^{n}, \nabla z_{1}\right\rangle-\left\langle f^{n}, z_{1}\right\rangle=0, \quad z_{1} \in H_{3}^{0}, \\
\left\langle Q^{n}, z_{2}\right\rangle-\left\langle a^{n} \partial_{t} \nabla U^{n}+b^{n} \nabla U^{n}, z_{2}\right\rangle=0, \quad z_{2} \in H_{3}^{0} .
\end{array}\right.
$$

Theorem 3.1: The solutions of (4) and (2) are equivalent, existent and unique. 
Proof: From the Equation (3), it is clear that the solution of (2) must be the solution of (4).

Let $\left\{\zeta_{l}: l=1,2, \cdots, 4 M N\right\}=\left\{\left(x_{i k}, y_{j l}\right), i=1, \cdots, M, j=1, \cdots, N, k, l=1,2\right\}$, $\left\{Z_{i}\right\}_{4 M N}$ be a group base of $H_{3}^{0}$. Thereupon $\forall U^{n}(x, y) \in H_{3}^{0}$ can be expressed as $U^{n}(x, y)=\sum_{i=1}^{4 M N} \beta_{i}^{n} Z_{i}(x, y)$. So (2) and (4) can be written in the form as follows

$$
\begin{gathered}
F \beta^{n}+G \beta^{n-1}=R, C \beta^{n}+D \beta^{n-1}=S, \\
F=\left(F_{i j}\right)_{4 M N \times 4 M N}, F_{i j}=Z_{j}\left(\zeta_{i}\right)-a^{n} \Delta Z_{j}\left(\zeta_{i}\right)-b^{n} \Delta t \Delta Z_{j}\left(\zeta_{i}\right), \\
C=\left(C_{i j}\right)_{4 M N \times 4 M N}, C_{i j}=\left\langle Z_{j}\left(\zeta_{i}\right), Z_{i}\right\rangle-a^{n}\left\langle\Delta Z_{j}\left(\zeta_{i}\right), Z_{i}\right\rangle-b^{n} \Delta t\left\langle\Delta Z_{j}\left(\zeta_{i}\right), Z_{i}\right\rangle,
\end{gathered}
$$

where $G, D$ are both matrixs of $4 M N \times 4 M N$ and $R, S$ are both vectors of $4 M N$. Obviously the solution of equation $F \tau=0$ must be satisfied the equation $C \tau=0$, when $\tau$ is a vectors of $4 M N$. So $F$ is nonsingular when $C$ is nonsingular. Then the solutions of (2) and (4) are unique. To get the existence and uniqueness, we just need to prove $A=\left(A_{i j}\right)_{4 M N \times 4 M N}$ where $A_{i j}=\left\langle Z_{j}\left(\zeta_{i}\right)-a \Delta Z_{j}\left(\zeta_{i}\right), Z_{i}\right\rangle$ is nonsingular when $\Delta t$ is sufficiently small. And the nonsingularity of $A$ has been proved [8] in. Thus the theorem is proved.

Next we will need to analyse the error estimate of (4).

\section{Error Estimate}

Define interpolation operators $\left(P_{1}, P_{2}\right)$ which satisfied the following conditions

$$
\begin{gathered}
W=P_{1} u, v=W-U, \eta=u-W, V=P_{2} q, w=V-Q, \xi=q-V, \\
\left\langle\nabla\left(q^{n}-V^{n}\right), z\right\rangle=0, \forall z \in H_{3}, \\
\left\langle a^{n} \nabla\left(u_{t}^{n}-W_{t}^{n}\right)+b^{n} \nabla\left(u^{n}-W^{n}\right), \nabla z\right\rangle=0, \forall z \in H_{3},
\end{gathered}
$$

i.e., $u-U=v+\eta, q-Q=w+\xi$. Now we can get the error equations

$$
\left\{\begin{array}{l}
\left\langle\eta_{t}^{n}, z_{1}\right\rangle+\left\langle\partial_{t} v^{n}, z_{1}\right\rangle+\left\langle r^{n}, z_{1}\right\rangle+\left\langle\xi^{n}+w^{n}, \nabla z_{1}\right\rangle=0, \quad z_{1} \in H_{3}^{0}, \\
\left\langle\xi^{n}+w^{n}, z_{2}\right\rangle-\left\langle a^{n}\left(\nabla r^{n}+\partial_{t} \nabla v^{n}\right), z_{2}\right\rangle-\left\langle b^{n} \nabla v^{n}, z_{2}\right\rangle=0, \quad z_{2} \in H_{3}^{0} .
\end{array}\right.
$$

where $r^{n}=W_{t}^{n}-\partial_{t} W^{n}, \nabla r^{n}=\nabla W_{t}^{n}-\partial_{t} \nabla W^{n}$. Then there is the theorem as follows.

Theorem 4.1: If $u(x, y)$ is the accurate solution of (1), $U(x, y)$ is the solution of the orthogonal collocation method, and $u(x, y)$ satisfies the condition [4] [7] $u \in L^{\infty}\left(0, T ; H^{6}(\Omega)\right) \cap L^{\infty}\left(0, T ; H^{6}(\Omega)\right), \quad u_{t} \in L^{\infty}\left(0, T ; H^{6}(\Omega)\right)$, then there is the error estimate as follows

$$
\left\|u^{n}-U^{n}\right\| \leq O\left(h^{4}+\Delta t\right),\left\|q^{n}-Q^{n}\right\| \leq O\left(h^{4}+\Delta t\right) .
$$

Proof: First, it is clearly for $r^{n}, \nabla r^{n}$ that

$$
\left\|r^{n}\right\|^{2} \leq C \Delta t \int_{t_{n-1}}^{t_{n}}\left\|W_{t t}\right\|^{2} \mathrm{~d} s,\left\|\nabla r^{n}\right\|^{2} \leq C \Delta t \int_{t_{n-1}}^{t_{n}}\left\|\nabla W_{t t}\right\|^{2} \mathrm{~d} s .
$$

Then let $z_{1}=v^{n}, z_{2}=\nabla v^{n}$ in (5), the equations 


$$
\left\{\begin{array}{l}
\left\langle\eta_{t}^{n}, v^{n}\right\rangle+\left\langle\partial_{t} v^{n}, v^{n}\right\rangle+\left\langle r^{n}, v^{n}\right\rangle+\left\langle\xi^{n}+w^{n}, \nabla v^{n}\right\rangle=0, \\
\left\langle\xi^{n}+w^{n}, \nabla v^{n}\right\rangle-\left\langle a^{n}\left(\nabla r^{n}+\partial_{t} \nabla v^{n}\right), \nabla v^{n}\right\rangle-\left\langle b^{n} \nabla v^{n}, \nabla v^{n}\right\rangle=0
\end{array}\right.
$$

can be got. It is easily calculated to see that

$$
a^{n}\left\langle\partial_{t} \nabla v^{n}, \nabla v^{n}\right\rangle+\left\langle\partial_{t} v^{n}, v^{n}\right\rangle=-a^{n}\left\langle\nabla r^{n}, \nabla v^{n}\right\rangle-b^{n}\left\langle\nabla v^{n}, \nabla v^{n}\right\rangle-\left\langle r^{n}, v^{n}\right\rangle-\left\langle\eta_{t}^{n}, v^{n}\right\rangle .
$$

Then through the Cauchy inequality, $\varepsilon$-inequality and $\left\langle\partial_{t} v^{n}, v^{n}\right\rangle \geq \frac{1}{2} \partial_{t}\left\|v^{n}\right\| \|^{2}$, and the functions $a$ and $b$ are bounded, it leads to the inequality

$$
\begin{aligned}
& \frac{1}{2} \partial_{t}\left\|\nabla v^{n}\right\|^{2}+\frac{1}{2} \partial_{t}\left\|v^{n}\right\|^{2} \\
& \leq K\left\|\nabla r^{n}\right\|^{2}+\varepsilon\left\|\nabla v^{n}\right\|\left\|^{2}+\right\| \nabla v^{n}\left\|^{2}+K\right\| r^{n} \|^{2} \\
& +\varepsilon\left\|v^{n}\right\|^{2}+K\left\|\eta_{t}^{n}\right\|^{2}+\varepsilon\left\|v^{n}\right\|^{2} .
\end{aligned}
$$

The coefficients $K, C$ both have nothing to do with $h, \Delta t$ in the upper equation and following proof. Add the inequality (6) and make summation to the series sum from $n=1$ to $n$ and multiply $\Delta t$. Then

$$
\begin{aligned}
& \left\|\nabla v^{n}\right\|^{2}+\left\|v^{n}\right\|^{2} \\
& \leq K \Delta t \sum_{i=0}^{n}\left(\left\|\eta_{t}^{i}\right\|^{2}+\left\|\nabla v^{i}\right\|\left\|^{2}+\right\| v^{i} \|\left.\right|^{2}\right)+K \Delta t^{2} \int_{0}^{t_{n}}\left(\left\|\nabla W_{t t}\right\|^{2}+\left\|W_{t t}\right\|^{2}\right) \mathrm{d} s
\end{aligned}
$$

is obtained. So it follows from discrete Gronwall lemma that

$$
\left\|\nabla v^{n}\right\|^{2}+\left\|v^{n}\right\|^{2} \leq K \Delta t \sum_{i=0}^{n}\left(\left\|\eta_{t}^{i}\right\|^{2}\right)+K \Delta t^{2} \int_{0}^{t_{n}}\left(\left\|\nabla W_{t t}\right\|^{2}+\mid\left\|W_{t t}\right\|^{2}\right) \mathrm{d} s
$$

if $\Delta t$ is small enough.

Second, let $z_{1}=\partial_{t} v^{n}, z_{2}=\partial_{t} \nabla v^{n}$ in (5), the equations

$$
\left\{\begin{array}{l}
\left\langle\eta_{t}^{n}, \partial_{t} v^{n}\right\rangle+\left\langle\partial_{t} v^{n}, \partial_{t} v^{n}\right\rangle+\left\langle r^{n}, \partial_{t} v^{n}\right\rangle+\left\langle\xi^{n}+w^{n}, \partial_{t} \nabla v^{n}\right\rangle=0, \\
\left\langle\xi^{n}+w^{n}, \partial_{t} \nabla v^{n}\right\rangle-\left\langle a^{n}\left(\nabla r^{n}+\partial_{t} \nabla v^{n}\right), \partial_{t} \nabla v^{n}\right\rangle-\left\langle b^{n} \nabla v^{n}, \partial_{t} \nabla v^{n}\right\rangle=0,
\end{array}\right.
$$

can be got. It is easy to get

$$
\begin{aligned}
& a^{n}\left\langle\partial_{t} \nabla v^{n}, \partial_{t} \nabla v^{n}\right\rangle+\left\langle\partial_{t} v^{n}, \partial_{t} v^{n}\right\rangle \\
& =-a^{n}\left\langle\nabla r^{n}, \partial_{t} \nabla v^{n}\right\rangle-b^{n}\left\langle\nabla v^{n}, \partial_{t} \nabla v^{n}\right\rangle-\left\langle r^{n}, \partial_{t} v^{n}\right\rangle-\left\langle\eta_{t}^{n}, \partial_{t} v^{n}\right\rangle .
\end{aligned}
$$

Then through Cauchy inequality and $\varepsilon$-inequality, (6) and (7) it leads to the inequality

$$
\begin{aligned}
& \left\|\partial_{t} \nabla v^{n}\right\|^{2}+\left\|\partial_{t} v^{n}\right\| \|^{2} \\
& \leq K \mid\left\|\eta_{t}^{n}\right\|^{2}+K \Delta t \sum_{i=1}^{n}\left\|\eta_{t}^{i}\right\|^{2}+K \Delta t^{2} \int_{0}^{t_{n}}\left(\left\|\nabla W_{t t}\right\|^{2}+\left\|W_{t t}\right\|^{2}\right) \mathrm{d} s,
\end{aligned}
$$

if $\Delta t$ is sufficiently small.

At last, let $z_{2}=w^{n}$ in the second equation of (5), it can be expressed as $\left\langle\xi^{n}+w^{n}, w^{n}\right\rangle-\left\langle a^{n}\left(\nabla r^{n}+\partial_{t} \nabla v^{n}\right), w^{n}\right\rangle-\left\langle b^{n} \nabla v^{n}, w^{n}\right\rangle=0$. (7) and (8) implies that 


$$
\begin{aligned}
\left.\left\|w^{n}\right\|\right|^{2} \leq & \left.K\left\|\xi^{n}\right\|\right|^{2}+K\left\|\left|\| \eta _ { t } ^ { n } \| \left\|^{2}+K \Delta t \sum_{i=1}^{n}\left|\left\|\eta_{t}^{i} \mid\right\|^{2}\right.\right.\right.\right. \\
& +K \Delta t^{2} \int_{0}^{t_{n}}\left(\left.\left\|\nabla W_{t t}\right\|\right|^{2}+\left.\left\|W_{t t}\right\|\right|^{2}\right) \mathrm{d} s .
\end{aligned}
$$

The results

$$
\begin{aligned}
& \|\eta\| \leq C h^{4}\left(\sum_{i, j}\left\|u^{(4)}\right\|^{2}\right)^{\frac{1}{2}},\left\|\eta_{t}\right\| \leq C h^{4}\left(\sum_{i, j}\left\|u_{t}^{(4)}\right\|^{2}\right)^{\frac{1}{2}}, \\
& \|\xi\| \leq C h^{4}\left(\sum_{i, j}\left\|q^{(1)}\right\|^{2}\right)^{\frac{1}{2}},\left\|\xi_{t}\right\| \leq C h^{4}\left(\sum_{i, j}\left\|q_{t}^{(4)}\right\|^{2}\right)^{\frac{1}{2}},
\end{aligned}
$$

can be obtained from lemma 1.6 in [4], where $u$ is sufficiently smooth ( $C$ is a positive constant). Moreover (3) in [7] implies that $\forall f \in H_{3},\|f\| \leq\|f\| \leq C\|f\|$ is valid. So it follows from (7), (9) and (10) that

$$
\begin{aligned}
\left\|u^{n}-U^{n}\right\|^{2} & \leq\left\|\eta^{n}\right\|^{2}+K\left\|\eta_{t}\right\|^{2}+K \Delta t^{2} \int_{0}^{t_{n}}\left(\left\|\nabla W_{t t}\right\|^{2}+\left\|W_{t t}\right\|^{2}\right) \mathrm{d} s \leq K_{1} h^{8}+K_{1} \Delta t^{2}, \\
\left\|q^{n}-Q^{n}\right\|^{2} & \leq K\left\|\xi^{n}\right\|^{2}+K\left\|\eta_{t}^{n}\right\|^{2}+K\left\|\eta_{t}\right\|^{2}+K \Delta t^{2} \int_{0}^{t_{n}}\left(\left\|\nabla W_{t t}\right\|\left\|^{2}+\right\| W_{t t} \|^{2}\right) \mathrm{d} s \\
& \leq K_{2} h^{8}+K_{2} \Delta t^{2},
\end{aligned}
$$

where $K_{1}$ and $K_{2}$ are constants which have nothing to do with $h$ and $\eta_{t}$. Thus the theorem is proved.

\section{Acknowledgements}

Sincere thanks to the Basic Subjects Fund and Science Foundations of China University of Petroleum (Beijing) (NO. 2462015YQ0604, NO. 2462015QZDX02).

\section{References}

[1] Wang, G. (2002) The Characteristic-Difference Method for a Nonlinear Sobolev Equation. Journal of Inner Mongolia Normal University, 31, 21-25.

[2] Guo, L. and Chen, H. (2006) $H^{\mathrm{l}}$-Galerkin Mixed Finite Element Method for the Sobolev Equation. Journal of System Science and Mathematical Science, 26, 301-314.

[3] Jiang, Z. and Chen, H. (2001) Error Estimates for Mixed Finite Element Method for Sobolev Equation. Northeastern Mathematical Hournal, 17, 301-314.

[4] Lu, T. (1994) The Finite Collocation Method for Parabolic Equation. Journal of Shandong University, 29, 266-272.

[5] Beck, J., Tempone, P. and Tamellini, F.N.L. (2012) On the Optiaml Polynomial Approximation of Stochastic PDEs by Galerkin and Collocation Methods. Mathematical Models \& Methods in Applied Sciences, 22, 199-218. https://doi.org/10.1142/S0218202512500236

[6] Parand, K. and Nikarya, M. (2017) A Numerical Method to Solve the 1D and the 2D Reaction Diffusion Equation Based on Bessel Functions and Jacobian Free Newton-Krylov Sub-Space Methods. European Physical Journal-Plus, 132, 496. https://doi.org/10.1140/epjp/i2017-11787-x

[7] Fernandes, R.I. and Fairweather, G. (1993) Analysis of Alternating Direction Collocation Methods for Parabolic and Hyperbolic Problems in Two Space Variables. Numerical Methods Partial Differential Equations, 9, 191-211. 
N. Ma et al.

https://doi.org/10.1002/num.1690090207

[8] Bi, H. (2010) Collocation Methods for Burgers Equation. Mathematical Mechanics and Mathematical Physics, 1, 105-112. 